\title{
Information Processing in Nanoscale Arrays: DNA Assembly, Molecular Devices, Nano-Array Architectures
}

\author{
Richard A. Kiehl \\ Department of Electrical \& Computer Engineering, University of Minnesota \\ 200 Union St. S.E., EE/CS 4-174, Minneapolis, Minn. 55455 \\ +01612 625-8073 \\ kiehl@ece.umn.edu
}

\begin{abstract}
Arrays of simple, nanoscale components are promising for future information processing circuitry. Large arrays could provide high functionality by exploiting the nonlinear dynamics of locally connected components, while circumventing nanoscale power dissipation and interconnect limits. To realize such a paradigm shift, however, many new physical design and system-level issues must be addressed. Here, I will describe 1) results on DNAdirected assembly of components in 2D arrays, 2) the search for electrically active molecular components and 3) schemes for information processing in arrays of simple nanoscale components.
\end{abstract}

\section{Keywords}

Nanoelectronics, self-assembly, molecular devices, nanoarchitecture.

\section{INTRODUCTION}

While the progress in electronics thus far has relied on the scaling of transistors and an increasingly complex maze of interconnecting wires, future advances in circuitry will require mating CMOS technology with new types of fabrication technologies, device principles, and circuit architectures. In particular, power dissipation limits and the interconnect bottleneck at high integration levels, coupled with the inherently low drive capability of small devices, will mandate the use of relatively simple, ultrasmall devices laid out in highly regular arrays and interconnected predominantly locally - all done with nanometer-scale precision.

Such a paradigm shift would require the convergence of radically new approaches in several different areas[2]. A versatile process for the self-assembly of nanocomponents into regular 2D arrays with programmable component spacing and precise registry would enable the manufacturing of circuits. Electrically active metal-molecule-metal (MmM) junctions could provide simple two-terminal components that could be scaled to single-molecule dimensions for assembly into dense arrays. Large arrays of such components could provide information processing at high functionality by exploiting the nonlinear dynamics of locally connected networks, thereby circumventing nanoscale power

\footnotetext{
Permission to make digital or hard copies of all or part of this work for personal or classroom use is granted without fee provided that copies are not made or distributed for profit or commercial advantage and that copies bear this notice and the full citation on the first page. To copy otherwise, to republish, to post on servers or to redistribute to lists, requires prior specific permission and/or a fee.

ICCAD'06, November 5-9, 2006, San Jose, CA

Copyright 2006 ACM 1-59593-389-1/06/0011 ...\$5.00
}

dissipation and interconnect limits. Here, I discuss our work in these three emerging areas of nanoelectronics research.
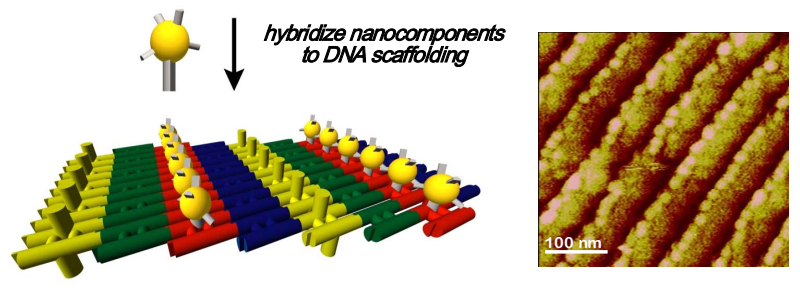

Figure 1. (Left) Schematic illustrating the self-assembly of metallic nanocomponent arrays by in situ hybridization to DNA scaffolding. (Right) AFM image of assembled arrays of 5-nm Au nanocomponents with intercomponent and inter-row spacings of 10 and $64 \mathrm{~nm}$, respectively.

\section{DNA-DIRECTED ASSEMBLY OF 2D NANOCOMPONENT ARRAYS}

A versatile process for manufacturing $2 \mathrm{D}$ nanocomponent arrays with programmable component spacing and precise registry would enable the development of nanoelectronic circuits based on regular arrays of devices. In addition to such conventional circuits as random access memories and programmable logic arrays, cellular nonlinear networks are of particular interest.[8] DNA provides a basis for self-assembly that can be programmed by base sequence design and offers a precision commensurate with its $\sim 0.3-\mathrm{nm}$ nucleotide building block. As shown in Fig. 1 and described in its legend, we have demonstrated precise selfassembly of 5-nm metallic nanocomponents into a 2D array by in situ hybridization of the nanocomponents to a 2D DNA scaffolding on a surface [4]. We have also demonstrated sequence-encoded self-assembly of different types of nanocomponents to selected sites on the scaffolding[7]. The programmability and precision of this approach could lead to a versatile manufacturing process for nanoelectronics.

\section{THE SEARCH FOR ELECTRICALLY ACTIVE MOLECULAR DEVICES}

Electrically active metal-molecule-metal (MmM) junctions are of interest for electronics at the nanoscale, where extreme geometric constraints strongly favor two-terminal diodes over three-terminal transistors. A variety of $\mathrm{MmM}$ junctions that exhibit negative differential resistance have been reported[3]. The mechanism 
behind the observed NDR has been the subject of much speculation. We have carried out a study of MmM junctions that exhibit stable NDR characteristics over many bias sweeps, thereby permitting a systematic study of electrical behavior[5]. Although the NDR peak position and height are strongly dependent on sweep rate, the amount of charge that flows during the NDR branch $Q_{F}$ is not. As shown in Fig. 2, the currentvoltage characteristic varies exponentially below and above the NDR branch, but at rates corresponding to different effective tunneling barriers $\varphi_{B 1}$ and $\varphi_{B 2}$. The cyclic characteristics retrace, except within the NDR region, and thus are hysteretic. Collectively, these observations reveal that the NDR in these experiments is caused by charge storage (oxidation or reduction) within the junction that acts to increase the effective tunneling barrier. Thus, the NDR is a dynamic effect and not the result of a resonant tunneling mechanism. Further analysis is needed to determine whether the junctions are locally active in the NDR region and, hence, could offer useful electronic functions.[1] More generally, these results are useful for developing a detailed physical understanding of these effects, which is needed for the synthesis of molecular systems with desired electrical properties.

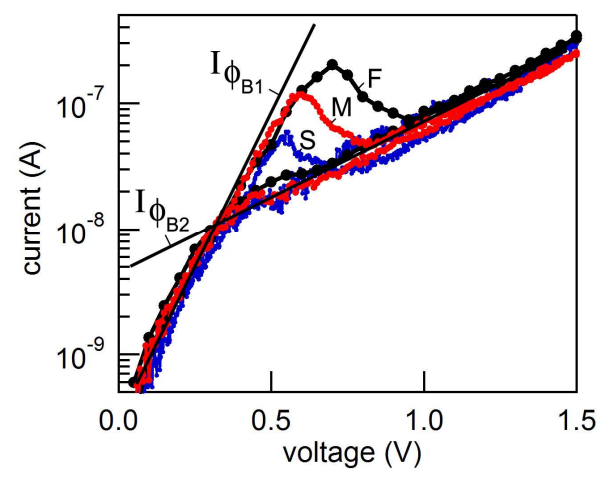

Figure 2 - Cyclic I-V characteristics for three different sweep rates. Each continuous cycle shows a current peak in the forward-sweep direction and no peak in reverse .

\section{INFORMATION PROCESSING IN NANO-ARRAYS}

Nanoscale arrays of simple nonlinear devices would open the door to powerful information processing systems that circumvent the interconnect bottleneck by exploiting the nonlinear evolution of phase dynamics in locally connected arrays[6,8]. Logic states in such arrays can be defined by the electrical phase of a dynamic physical process, such as electron tunneling in ultra-small junctions or in molecules. These processes produce impulsive "neuron-like" waveforms that can be coupled to nearest neighbors in a 2D (or possibly 3D) array. Input data can be represented by the distribution of dc bias level, initial charge, or coupling strength across the array. Information processing is realized through the nonlinear dynamics produced by interactions between the elements in the array, rather than through Boolean operations. The dynamics give rise to an evolution of complex twodimensional patterns in the phase-state across the array and represent a computation on the input data. Simulated results for a network with uniform electrostatic coupling between each element and its four nearest neighbors are shown in Fig. 3. The results demonstrate the generation of complex patterns and repeating sequences of patterns and the capability for performing some simple image processing tasks. Appropriate design of the $2 \mathrm{D}$ coupling distribution within the array could lead to capabilities for performing higher-level image processing tasks and, possibly, more general information processing functions.

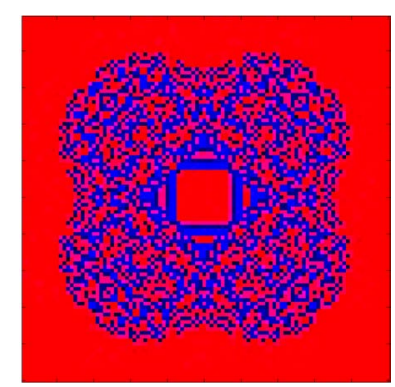

Figure 3 - Phase-state map for 100 x 100 network of locally connected integrate-and-fire elements, illustrating the generation of complex patterns.

\section{CONCLUSION}

While work in DNA-directed assembly, active molecular devices, and nano-array architectures is still in its infancy, these approaches could lead to powerful information processing systems that exploit massive parallelism in simple, highly scalable nano-architectures. The realization of this paradigm shift will raise a host of non-traditional physical design and system-level issues, which must be addressed along the way.

The author would like to acknowledge his collaborators and support by NSF and MARCO/FENA.

\section{REFERENCES}

1. Chua, L.O. Nonlinear circuit foundations for nanodevices, part I: The four-element torus. Proceedings of the IEEE, 91 (11). 1830-1859.

2. Kiehl, R.A. Nanoparticle electronic architectures assembled by DNA. J. Nanopart. Res., 2. 331-332.

3. Le, J.D., He, Y., Hoye, T.R., Mead, C.C. and Kiehl, R.A. Negative differential resistance in a bilayer molecular junction. Applied Physics Letters, 83 (26). 5518-5520.

4. Le, J.D., Pinto, Y., Seeman, N.C., Musier-Forsyth, K., Taton, T.A. and Kiehl, R.A. DNA-templated self-assembly of metallic nanocomponent arrays on a surface. Nano Lett., 4 (12). 2343-2347.

5. Le, J.D., Pinto, Y., Seeman, N.C., Musier-Forsyth, K., Taton, T.A. and Kiehl, R.A. DNA-templated self-assembly of metallic nanocomponent arrays on a surface. Nano Letters, 4 (12). 2343-2347.

6. Li, T. and Kiehl, R.A. Operating regimes for multi-valued single-electron tunneling logic. J. Appl. Phys.

7. Pinto, Y.Y., Le, J.D., Seeman, N.C., Musier-Forsyth, K., Taton, T.A. and Kiehl, R.A. Sequence-encoded selfassembly of multiple-nanocomponent arrays by 2D DNA scaffolding. Nano Lett., 5 (12). 2399 -2402.

8. Yang, T., Kiehl, R.A. and Chua, L.O. Tunneling phase logic cellular nonlinear networks. International Journal of Bifurcation \& Chaos in Applied Sciences \& Engineering, 11 (12). 2895-2911. 